\title{
Rapid and Accurate Identification of Escherichia coli K-12 Strains
}

\author{
PETER KUHNERT, ${ }^{*}$ JACQUES NICOLET, AND JOACHIM FREY \\ Institute of Veterinary Bacteriology, University of Bern, CH-3012 Bern, Switzerland
}

Received 24 May 1995/Accepted 31 August 1995

\begin{abstract}
A specific PCR for the identification of K-12 strains, based on the genetic structure of the O-antigen gene cluster $(r f b)$ of Escherichia coli $\mathrm{K}-12$, is described. The assay clearly differentiates $E$. coli K-12-derived strains from other $E$. coli strains used in the laboratory or isolated from human and animal clinical specimens, from food, or from environmental samples. Moreover, lineages of K-12 strains can be distinguished with a second PCR based on the same gene cluster. The method presents a useful tool in identifying K-12 for monitoring strains which are used as biologically safe vehicles in biotechnological research, development, and production processes.
\end{abstract}

Escherichia coli $\mathrm{K}-12$ strains are by far the most frequently used host strains in gene cloning experiments, since they have the following advantages: (i) they represent the genetically best understood living organism, (ii) they are easily modified by many genetic methods, and (iii) they are classified as biologically safe vehicles for the propagation of many efficient gene cloning and expression vectors in all major national and international guidelines on biological safety for work with recombinant DNA technology. An increasing number of different strains, both K-12 derivatives and other $E$. coli strains, are ever emerging for use in biotechnological research and development. The broad genetic and phenotypic ranges of the varieties deduced from K-12 and other E. coli strains cause major problems in identifying their correct origins. Entire pedigrees (1) would therefore be required for clear identification of strains. A complete description of bacterial lineages, however, cannot be given in many cases. This can cause severe problems in the interpretation of experimental data and in particular in biosafety assessments, especially since no character common to $E$. coli $\mathrm{K}-12$ strains is known to clearly differentiate them from other, apparently very similar, E. coli strains.

The wild-type strain of $E$. coli $\mathrm{K}-12$ was isolated from the feces of a convalescent diphtheria patient in 1922 at Stanford University. Subcultures and derivatives of this strain were first reported in 1944 (3). Since then, the strain has been intensively used and mutagenized in many laboratories, and no case of disease has ever been reported to be caused by it. It was also shown that K-12 strains are unable to colonize the human gut (9). Moreover, K-12 strains are devoid of all known E. coli virulence genes (6). The K-12 lineage is therefore considered to be a prototype of safe and nonpathogenic bacterial strains. Hence, an accurate and rapid method to discriminate $E$. coli $\mathrm{K}-12$ from other $E$. coli strains is needed as a standard tool in biological safety procedures.

E. coli K-12 strains are rough, apparently lacking the $\mathrm{O}$ antigen which is part of the lipopolysaccharide and which is encoded by the $r f b$ gene cluster. Liu and Reeves (5) recently showed that the lack of $\mathrm{O}$ antigen in some $\mathrm{K}-12$ derivative strains is due to a mutation $(r f b-50)$ within the $r f b$ cluster which inactivates the rhamnose transferase, a key enzyme in the $\mathrm{O}$ antigen biosynthesis. The $r f b-50$ mutation is characterized by

* Corresponding author. Mailing address: Institute of Veterinary Bacteriology, Laenggass-Str. 122, CH-3012 Bern, Switzerland. Phone: 41-31-6312369. Fax: 41-31-6312634. Electronic mail address: Kuhnert $@$ vbi.unibe.ch. an IS5 insertion that is located within the last gene (orf264, also named orf11 or orf5) of the $r f b$ cluster encoding the rhamnose transferase $(5,10)$ (Fig. 1). This mutation can be complemented with the wild-type sequences from the ancestral K-12 strain WG1 as well as from Shigella flexneri $(5,11)$. While most $\mathrm{K}-12$ derivatives seem to carry the $r f b-50$ mutation, the ancestral strain WG1, which is assumed to be the K-12 wild-type strain, contains a functional orf264 (5). However, WG1 carries a mutation $(r f b-51)$ in another gene of the $r f b$ cluster. This $r f b-51$ mutation is not present within most K-12 derivatives and might have occurred as an independent event (5).

A variation of the $r f b-50$ mutation characteristic for $\mathrm{K}-12$ derivatives is described for $\mathrm{K}-12$ strain AB311. This strain contains a deletion of part of orf264 upstream IS5 and a substitution by a sequence (sqx) of unknown function (5). We developed two PCRs based on the DNA sequences of orf 264 and IS5. With these reactions, we detected the presence of orf 264; the IS5-induced mutation $r f b-50$, which is characteristic of K-12 derivatives; and the variation of $r f b-50$ which distinguishes the AB311 side lineage of $\mathrm{K}-12$. We present data from screening of a large variety of $E$. coli K-12 strains as well as other $E$. coli strains used in laboratories or isolated from humans, animals, or the environment.

Sample preparation for PCR. The bacterial strains were obtained from different origins including the $E$. coli Genetic Stock Center, Yale University (kindly provided by B. Bachmann), the collection of L. Caro (University of Geneva, Geneva, Switzerland), commercial suppliers, and our own collection. For the human pathogens, genomic DNA from strains of the Salmonella Reference Bank and from the collection of the Swiss Reference Laboratory for Foodborne Diseases, Bern, Switzerland, was used (kindly provided by A. Burnens). Environmental $E$. coli isolates were obtained from the Group for Environmental Hygiene, University of Zurich (kindly provided by A. Metzler), and from the Institute for Medical Microbiology, University of Hannover (kindly provided by M. Frosch).

All E. coli strains were grown overnight on Luria-Bertani plates containing, per liter, $10 \mathrm{~g}$ of Bacto Tryptone (Difco), 5 $\mathrm{g}$ of yeast extract (Difco), $5 \mathrm{~g}$ of $\mathrm{NaCl}, 2 \mathrm{ml}$ of $1 \mathrm{M} \mathrm{NaOH}$, and $15 \mathrm{~g}$ of agar (Difco). Three to five colonies were lysed in $450 \mu \mathrm{l}$ of lysis buffer $(100 \mathrm{mM}$ Tris- $\mathrm{HCl}[\mathrm{pH} 8.5], 0.05 \%$ Tween 20 , and $240 \mu \mathrm{g}$ of proteinase $\mathrm{K}$ per $\mathrm{ml}$ ). Samples were incubated for $1 \mathrm{~h}$ at $60^{\circ} \mathrm{C}$ and then heated to $97^{\circ} \mathrm{C}$ for $15 \mathrm{~min}$ in order to inactivate proteinase $\mathrm{K}$.

Oligonucleotide primers and PCR assay. On the basis of the report of Liu and Reeves (5) describing the $r f b-50$ mutation, we 


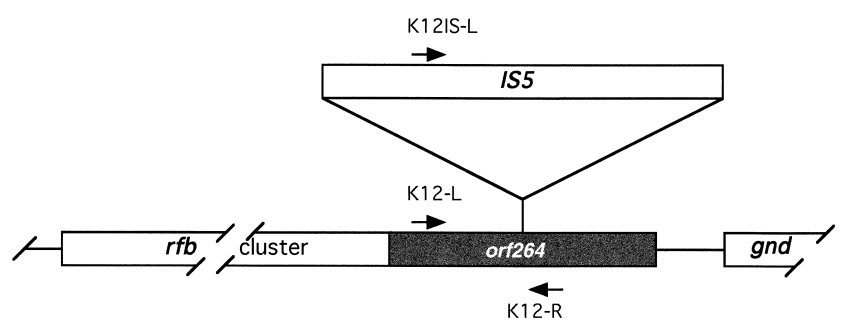

$200 \mathrm{bp}$

FIG. 1. Map of the $r f b$ cluster and region analyzed in this study. The $r f b$ cluster is located $5^{\prime}$ to the 6-phosphogluconate dehydrogenase gene (gnd). Boxes represent genes. The entire $r f b$ cluster extends over more than $10 \mathrm{~kb}$ and contains 11 genes. The gene located at the $3^{\prime}$ end of the cluster, orf 264 (shaded box), encodes the rhamnose transferase. It harbors an IS5 sequence in most K-12 strains specifying the $r f b-50$ mutation. Arrows indicate the locations of primers used for PCR.

selected two oligonucleotide primer sets to amplify a region of orf264. One pair of primers is dependent on the complete gene orf 264 and consists of primers K12-R (5'-ATCCTGCGCACC AATCAACAA-3') (nucleotides [nt] 508 to 488 on orf264) and K12-L (5'-TTCCCACGGACATGAAGACTACA-3') (nt 21 to 43 on orf264). The second pair includes primers K12-R and K12IS-L (5'-CGCGATGGAAGATGCTCTGTA-3') (nt 293 to 313 on IS5) and is dependent on the $3^{\prime}$ end of orf 264 and the presence of IS5 (Fig. 1).

In order to have an internal technical control for the PCRs, we synthesized primers that amplify a segment of the pal gene encoding the peptidoglycan-associated lipoprotein (2), which is conserved in $E$. coli and closely related bacteria. The primers are ECPAL-L (5'-GGCAATTGCGGCATGTTCTTCC-3') (nt 50 to 71 on $p a l$ ) and ECPAL-R (5'-CCGCGTGACCTTCT ACGGTGAC-3') (nt 328 to 307 on pal). Primer synthesis was done at Microsynth, Balgach, Switzerland.

PCR was performed with either a PE9600 or PE2400 automated thermocycler with MicroAmp tubes (Perkin-Elmer Cetus, Norwalk, Conn.). The reaction was carried out in a $50-\mu l$ volume containing $5 \mu \mathrm{l}$ of $10 \times$ PCR buffer (supplied with Taq DNA polymerase), 20 pmol of primer (each), $1 \mathrm{mM}$ deoxynucleoside triphosphate, $2.5 \mathrm{U}$ of Taq DNA polymerase (Boehringer, Mannheim, Germany), and $1 \mu$ l of bacterial lysate or DNA. PCR conditions were as follows: $3 \mathrm{~min}$ at $94^{\circ} \mathrm{C}$ followed by 35 cycles at $94^{\circ} \mathrm{C}$ for $30 \mathrm{~s}, 60^{\circ} \mathrm{C}$ for $30 \mathrm{~s}$, and $72^{\circ} \mathrm{C}$ for $1.5 \mathrm{~min}$. PCR products were analyzed on a $1 \%$ agarose gel (Sigma). Results from a few strains giving the typical patterns are shown in Fig. 2.

Sampling of $E$. coli strains and related enteric bacteria. Three major groups of $E$. coli strains were analyzed: (i) K-12 derivatives, (ii) other E. coli laboratory strains not derived from K-12, and (iii) E. coli strains isolated from patients, food, and the environment. Since E. coli is phylogenetically closely related to Shigella and Salmonella spp. and since the genes for the biosynthesis of the $\mathrm{O}$ antigens of certain Shigella and Salmonella strains are closely related to the $r f b$ genes found in K-12 (11), we also included Shigella and Salmonella strains in our study. Tables 1 and 2 give the list of all strains investigated and the data obtained from their analysis.

All E. coli, Shigella, and Salmonella strains amplified a 0.28-kb fragment of the pal gene with the primer pair ECPAL-LECPAL-R, which we included in our analysis as control, indi- cating that the pal gene is conserved among enteric bacteria. Nonenteric, gram-negative, and gram-positive bacteria showed no signal in this assay.

Identification of $\boldsymbol{E}$. coli $\mathbf{K}-12$ derivatives. WG1 is assumed to be the wild-type strain of E. coli K-12, or at least the nearest known descendant from it. Strains 58 and 679 , both used by Gray and Tatum in the 1940s (3), are the most antecedentdocumented mutants derived from $E$. coli K-12 (1). Virtually all K-12 derivative strains which are currently used as laboratory strains for gene cloning and expression are supposed to originate from these two strains, although the pedigree (1) does not enumerate many strains which are frequently used in gene cloning. In our K-12-specific PCR assay with oligonucleotide primers K12IS-L and K12-R (Fig. 1), all 39 analyzed K-12 derivatives including strains 58 and 679 revealed the $0.97-\mathrm{kb}$ band from amplification of the orf264-IS5 junction, showing that they all contain the $r f b-50$ mutation (Table 1 ). This set of strains contained known K-12 derivatives described in the pedigree (1) and commonly used hosts for gene cloning (7). This supports the data of the pedigree presented by Bachmann (1), indicating that all currently used $E$. coli K-12 strains are descendants from strain 58 or 679 or a very close relative of them. The PCR clearly distinguished the K-12 derivative strains from the supposed wild-type strain WG1, as well as from the related $E$. coli $\mathrm{O} 16: \mathrm{K} 1: \mathrm{H}$ and from all other strains of E. coli, Shigella, and Salmonella used in this study of different origins which lack the $0.97-\mathrm{kb}$ fragment of the IS5-orf264 junction (Tables 1 and 2).

Phylogenetic relationship of K-12 strains. A complementary PCR with the oligonucleotide primer pair K12-L-K12-R was designed to amplify the segment of orf 264 flanking the insertion locus of IS5 in K-12 derivative strains (Fig. 1). This PCR resulted in a 1.69-kb fragment from amplification of part of orf 264 and IS5 in all K-12 derivatives except in strains AB311

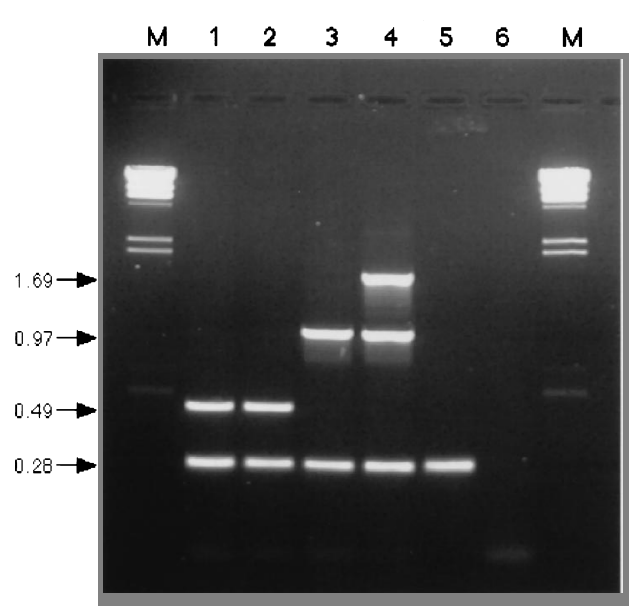

FIG. 2. PCR results for selected strains showing all observed patterns. Lanes: 1, E. coli $\mathrm{O} 16: \mathrm{K} 1: \mathrm{H} ; 2$, WG1 (assumed wild type); 3, AB311 (side lineage of K-12 derivatives); 4, C600 (representing the majority of $\mathrm{K}-12$ derivatives); 5 , S. flexneri (able to complement $r f b-50) ; 6, C$. perfringens (gram-positive control); $\mathrm{M}, \lambda$ HindIII marker with 23.1-, 9.4-, 6.6-, 4.4-, 2.3-, 2.0-, and 0.6-kb bands. Lanes 1 to 6 of the gel contain the amplification products of each of the three separate PCRs: specific 0.97-kb fragment of K-12 derivatives, resulting from amplification with primer pair K12IS-L-K12-R; amplification with primer pair K12-L-K12-R based on the orf 264 sequence, resulting in the 1.69 -kb band of practically all K-12 derivatives with the exception of strains AB311 and YN2980, or the 0.49-kb band present in E. coli serovar O16 and in strain WG1; and control amplification with primer pair ECPAL-L-ECPAL-R, resulting in a 280 -bp band with any E. coli or related enteric species. Aliquots of $2.5 \mu \mathrm{l}$ of the orf264-specific reaction mixtures (the first two above) and $5 \mu \mathrm{l}$ of the pal-specific reaction mixture (the last one above) were loaded on the same slot on a $1 \%$ agarose gel. 
TABLE 1. Laboratory bacterial strains used in this study and PCR results with the primer sets K12IS-L-K12-R, K12-L-K12-R, and ECPAL-L-ECPAL-R

\begin{tabular}{|c|c|c|c|c|c|c|c|}
\hline \multirow{2}{*}{ Strain no. } & \multirow{2}{*}{$\begin{array}{c}\text { Strain } \\
\text { designation }\end{array}$} & \multirow{2}{*}{ Name } & \multirow{2}{*}{ Origin or collection } & \multirow{2}{*}{$\begin{array}{l}\text { K12IS-L-K12-R, } \\
969 \text { bp }\end{array}$} & \multicolumn{2}{|c|}{ K12-L-K12-R } & \multirow{2}{*}{$\begin{array}{l}\text { ECPAL-L-ECPAL-R, } \\
278 \text { bp }\end{array}$} \\
\hline & & & & & $1,687 \mathrm{bp}$ & 488 bp & \\
\hline \multicolumn{8}{|c|}{$\begin{array}{l}\text { Ancestral } E \text {. coli } \mathrm{K}-12 \\
\text { strains }\end{array}$} \\
\hline CGSC 5073 & WG1 & K-12 & Wisconsin strain (wt) $)^{a, b}$ & - & - & + & + \\
\hline CGSC 5587 & 58 & $\mathrm{~K}-12$ & Stanford strain $(\text { bio- } 1)^{b}$ & + & + & - & + \\
\hline CGSC 5588 & 679 & $\mathrm{~K}-12$ & Stanford strain $(t h r-1)^{b}$ & + & + & - & + \\
\hline \multicolumn{8}{|c|}{ E. coli $\mathrm{K}-12$ derivatives } \\
\hline JF1512 & MB408 & K-12 & Laboratory strain & + & + & - & + \\
\hline JF1513 & AG1 & $\mathrm{K}-12$ & Laboratory strain & + & + & - & + \\
\hline JF1514 & JM101 & $\mathrm{K}-12$ & Laboratory strain & + & + & - & + \\
\hline JF1515 & DH5 $\alpha$ & $\mathrm{K}-12$ & Laboratory strain & + & + & - & + \\
\hline JF1412 & DH5 $\alpha$ & $\mathrm{K}-12$ & Laboratory strain & + & + & - & + \\
\hline JF49 & C600 & $\mathrm{K}-12$ & Laboratory strain ${ }^{b}$ & + & + & - & + \\
\hline JF129 & W3110 & $\mathrm{K}-12$ & Laboratory strain ${ }^{b}$ & + & + & - & + \\
\hline JF172 & Hfr 3000 & $\mathrm{~K}-12$ & Laboratory strain ${ }^{b}$ & + & + & - & + \\
\hline JF273 & JM83 & K-12 & Laboratory strain & + & + & - & + \\
\hline JF520 & $5 \mathrm{~K}$ & $\mathrm{~K}-12$ & Laboratory strain & + & + & - & + \\
\hline JF553 & DH1 & $\mathrm{K}-12$ & Laboratory strain ${ }^{b}$ & + & + & - & + \\
\hline JF1464 & DH1 & $\mathrm{K}-12$ & Laboratory strain ${ }^{b}$ & + & + & - & + \\
\hline JF1078 & H1443 & $\mathrm{K}-12$ & Laboratory strain & + & + & - & + \\
\hline JF1208 & TG1 & K-12 & Laboratory strain & + & + & - & + \\
\hline JF1224 & DP50 & $\mathrm{K}-12$ & Laboratory strain & + & + & - & + \\
\hline JF1427 & $?$ & $\mathrm{~K}-12$ & Laboratory strain & + & + & - & + \\
\hline JF1501 & W945 & $\mathrm{K}-12$ & Laboratory strain ${ }^{b}$ & + & + & - & + \\
\hline JF1502 & PA309 & $\mathrm{K}-12$ & Laboratory strain ${ }^{b}$ & + & + & - & + \\
\hline JF1503 & $58-161$ & $\mathrm{~K}-12$ & Laboratory strain ${ }^{b}$ & + & + & - & + \\
\hline JF1507 & P678 & $\mathrm{K}-12$ & Laboratory strain ${ }^{b}$ & + & + & - & + \\
\hline HB101 & HB101 & K-12/B hybrid & Laboratory strain & + & + & - & + \\
\hline JF554 & XL1-Blue & $\mathrm{K}-12$ & Stratagene ${ }^{c}$ & + & + & - & + \\
\hline JF1223 & XLOLR & K-12 & Stratagene & + & + & - & + \\
\hline JF980 & SURE & $\mathrm{K}-12$ & Stratagene & + & + & - & + \\
\hline JF1066 & HMS 174 & $\mathrm{~K}-12$ & Novagen $^{d}$ & + & + & - & + \\
\hline JF1069 & HMS 174 & $\mathrm{~K}-12$ & Novagen & + & + & - & + \\
\hline JF1392 & YN2980 & K-12 & Laboratory strain & + & - & - & + \\
\hline CGSC 311 & AB311 & $\mathrm{K}-12$ & Laboratory $\operatorname{strain}^{b}$ & + & - & - & + \\
\hline CGSC 5357 & Hfr $3000 \times 74$ & $\mathrm{~K}-12$ & Laboratory strain ${ }^{b}$ & + & + & - & + \\
\hline CGSC 4488 & Cavalli Hfr & $\mathrm{K}-12$ & Laboratory strain ${ }^{b}$ & + & + & - & + \\
\hline CGSC 253 & W208 & $\mathrm{K}-12$ & Laboratory strain ${ }^{b}$ & + & + & - & + \\
\hline CGSC 284 & AB284 & $\mathrm{K}-12$ & Laboratory strain ${ }^{b}$ & + & + & - & + \\
\hline CGSC 4401 & EMG2 & $\mathrm{K}-12$ & Laboratory strain & + & + & - & + \\
\hline CGSC 5024 & W1485 & $\mathrm{K}-12$ & Laboratory strain ${ }^{b}$ & + & + & - & + \\
\hline CGSC 5037 & $\mathrm{Y} 10$ & K-12 & Laboratory strain $^{b}$ & + & + & - & + \\
\hline CGSC 5608 & WA704 & K-12 & Laboratory strain ${ }^{b}$ & + & + & - & + \\
\hline CGSC 6613 & JC9387 & $\mathrm{K}-12$ & Laboratory strain ${ }^{b}$ & + & + & - & + \\
\hline \multicolumn{8}{|c|}{$\begin{array}{l}\text { E. coli laboratory strains } \\
\text { not derived from K-12 }\end{array}$} \\
\hline JF1504 & B & $\mathrm{B}$ & Laboratory strain & - & - & - & + \\
\hline JF1505 & B-3 & B & Laboratory strain & - & - & - & + \\
\hline JF1506 & $\mathrm{B} / \mathrm{R}$ & $\mathrm{B}$ & Laboratory strain & - & - & - & + \\
\hline JF1508 & $\mathrm{C}$ & $\mathrm{C}$ & Laboratory strain & - & - & - & + \\
\hline JF1509 & $\mathrm{C}$ & $\mathrm{C}$ & Laboratory strain & - & - & - & + \\
\hline JF1510 & $\mathrm{C}$ & $\mathrm{C}$ & Laboratory strain & - & - & - & + \\
\hline JF1500 & TOPP & $\mathrm{ND}^{e}$ & Stratagene & - & - & - & + \\
\hline JF702 & BL21 & B & Novagen & - & - & - & + \\
\hline JF703 & BL21 & B & Novagen & - & - & - & + \\
\hline
\end{tabular}

${ }^{a}$ wt, wild type.

${ }^{b}$ Reference 1.

${ }^{c}$ Stratagene, La Jolla, Calif.

${ }^{d}$ Novagen, Madison, Wis.

${ }^{e} \mathrm{ND}$, not determined.

and YN2980 (8) (Table 1), which showed no reaction product. Only the wild-type strain WG1 and $E$. coli serovar O16:K1:H showed a fragment of $0.49 \mathrm{~kb}$ in this PCR, which indicates that these two strains possess the original orf 264 gene without IS5 insertion. Previous results (5) showed that the E. coli deriva- tives EMG2 and W1485 (Table 1) could be complemented with the wild-type gene for rhamnose transferase (orf264) from WG1 for the expression of an intact $\mathrm{O}$ antigen which strongly reacted with anti-O16 antibodies but showed little cross-reaction with anti-O17 antibodies. While in our PCR E. coli serovar 
TABLE 2. Diagnostic and environmental isolates used in this study and PCR results ${ }^{a}$

\begin{tabular}{|c|c|c|c|c|c|c|c|}
\hline \multirow{2}{*}{ Strain no. } & \multirow{2}{*}{ Sp. } & \multirow{2}{*}{ Serovar } & \multirow{2}{*}{ Origin or collection } & \multirow{2}{*}{$\begin{array}{l}\text { K12IS-L-K12-R, } \\
969 \text { bp }\end{array}$} & \multicolumn{2}{|c|}{ K12-L-K12-R } & \multirow{2}{*}{$\begin{array}{c}\text { ECPAL-L-ECPAL-R } \\
278 \text { bp }\end{array}$} \\
\hline & & & & & $1,687 \mathrm{bp}$ & 488 bp & \\
\hline \multicolumn{8}{|l|}{ Gram-negative } \\
\hline NCTC 9016 & E. coli & $\mathrm{O} 16: \mathrm{K} 1: \mathrm{H}$ & Reference strain & - & - & + & + \\
\hline NCTC 9017 & E. coli & O17:K16:H18 & Reference strain & - & - & - & + \\
\hline JF1556 & E. coli & ND & Dairy product & - & - & - & + \\
\hline JF1557 & E. coli & ND & Dairy product & - & - & - & + \\
\hline JF1558 & E. coli & ND & Dairy product & - & - & - & + \\
\hline JF1559 & E. coli & ND & Dairy product & - & - & - & + \\
\hline JF1560 & E. coli & ND & Dairy product & - & - & - & + \\
\hline JF1561 & E. coli & ND & Dairy product & - & - & - & + \\
\hline JF1564 & E. coli & ND & Environment (water) & - & - & - & + \\
\hline JF1584 & E. coli & ND & Environment (water) & - & - & - & + \\
\hline JF1585 & E. coli & ND & Environment (water) & - & - & - & + \\
\hline JF1586 & E. coli & ND & Environment (water) & - & - & - & + \\
\hline JF1587 & E. coli & ND & Environment (water) & - & - & - & + \\
\hline JF1565 & E. coli & O128:K67 & Human, dysenteric stool & - & - & - & + \\
\hline JF1566 & E. coli & O128:K67 & Human, dysenteric stool & - & - & - & + \\
\hline JF1567 & E. coli & O128:K67 & Human, dysenteric stool & - & - & - & + \\
\hline JF1568 & E. coli & O119:K69 & Human, dysenteric stool & - & - & - & + \\
\hline JF1569 & E. coli & O127:K63 & Human, dysenteric stool & - & - & - & + \\
\hline JF1570 & E. coli & $\mathrm{O} 125: \mathrm{K} 70$ & Human, dysenteric stool & - & - & - & + \\
\hline JF1572 & E. coli & $\mathrm{O} 111: \mathrm{K} 58$ & Human, dysenteric stool & - & - & - & + \\
\hline JF1573 & E. coli & $\mathrm{O} 125: \mathrm{K} 70$ & Human, dysenteric stool & - & - & - & + \\
\hline JF1574 & E. coli & O55:K59 & Human, dysenteric stool & - & - & - & + \\
\hline JF1260 & E. coli & ND & Bovine mastitis & - & - & - & + \\
\hline JF1278 & E. coli & ND & Bovine mastitis & - & - & - & + \\
\hline JF1266 & E. coli & ND & Bovine mastitis & - & - & - & + \\
\hline JF1300 & E. coli & ND & Bovine septicemia & - & - & - & + \\
\hline JF1410 & E. coli & K99 & Bovine diarrhea & - & - & - & + \\
\hline JF1275 & E. coli & ND & Red deer, septicemia & - & - & - & + \\
\hline JF1295 & E. coli & ND & Ape, septicemia & - & - & - & + \\
\hline JF1265 & E. coli (ETEEC) & O139:K82 & Porcine diarrhea & - & - & - & + \\
\hline JF1321 & E. coli (ETEEC) & $\mathrm{O} 139: \mathrm{K} 82$ & Porcine diarrhea & - & - & - & + \\
\hline JF1305 & E. coli (ETEEC) & O141:(H4) & Porcine diarrhea & - & - & - & + \\
\hline JF1369 & E. coli (ETEEC) & O141:(H4) & Porcine diarrhea & - & - & - & + \\
\hline JF1381 & E. coli (ETEEC) & O141:(H4) & Porcine diarrhea & - & - & - & + \\
\hline JF1276 & E. coli (ETEEC) & O147:H19:K88 & Porcine diarrhea & - & - & - & + \\
\hline NZ2956 & E. coli (VTEC) & O157 & Human, hemorrhagic colitis & - & - & - & + \\
\hline NZ4253 & E. coli (VTEC) & O157 & Human, hemorrhagic colitis & - & - & - & + \\
\hline E1630-91 & E. coli (EPEC) & ND & Human, infant diarrhea & - & - & - & + \\
\hline E. coli $\mathrm{O} 111$ & E. coli (EPEC) & ND & Human, infant diarrhea & - & - & - & + \\
\hline NZ3211-94 & E. coli (ETEC) & ND & Human, traveller's diarrhea & - & - & - & + \\
\hline NZ3213-94 & E. coli (ETEC) & ND & Human, traveller's diarrhea & - & - & - & + \\
\hline NZ1631-94 & Shigella sonnei & ND & Human, dysenteric stool & - & - & - & + \\
\hline NZ666-94 & S. sonnei & ND & Human, dysenteric stool & - & - & - & + \\
\hline NZ1475-94 & S. sonnei & ND & Human, dysenteric stool & - & - & - & + \\
\hline NZ425-94 & S. sonnei & ND & Human, dysenteric stool & - & - & - & + \\
\hline NZ879-95 & S. sonnei & ND & Human, dysenteric stool & - & - & - & + \\
\hline NZ1360-95 & S. sonnei & ND & Human, dysenteric stool & - & - & - & + \\
\hline NZ1403-94 & Shigella dysenteriae & ND & Human, dysenteric stool & - & - & - & + \\
\hline NZ4142-90 & S. dysenteriae 1 & ND & Human, dysenteric stool & - & - & - & + \\
\hline NZ4800-92 & S. dysenteriae 1 & ND & Human, dysenteric stool & - & - & - & + \\
\hline NZ4894-94 & S. dysenteriae 1 & ND & Human, dysenteric stool & - & - & - & + \\
\hline NZ329-94 & S. dysenteriae 2 & ND & Human, dysenteric stool & - & - & - & + \\
\hline NZ359-94 & S. dysenteriae 2 & ND & Human, dysenteric stool & - & - & - & + \\
\hline NZ1208-94 & S. flexneri & ND & Human, dysenteric stool & - & - & - & + \\
\hline NZ1679-94 & S. flexneri & ND & Human, dysenteric stool & - & - & - & + \\
\hline NZ233-94 & S. flexneri & ND & Human, dysenteric stool & - & - & - & + \\
\hline NZ936-94 & S. flexneri & ND & Human, dysenteric stool & - & - & - & + \\
\hline NZ816-94 & S. flexneri & ND & Human, dysenteric stool & - & - & - & + \\
\hline NZ6733-93 & Shigella boydii & ND & Human, dysenteric stool & - & - & - & + \\
\hline NZ2142-93 & S. boydii & ND & Human, dysenteric stool & - & - & - & + \\
\hline SARB 17 & Salmonella enterica & Enteritidis & Reference strain & - & - & - & + \\
\hline SARB 18 & S. enterica & Enteritidis & Reference strain & - & - & - & + \\
\hline ATCC 23564 & S. enterica LT2 & Typhimurium & Reference strain & - & - & - & + \\
\hline SARB 66 & S. enterica & Typhimurium & Reference strain & - & - & - & + \\
\hline SARB 65 & S. enterica & Typhimurium & Reference strain & - & - & - & + \\
\hline SARB 27 & S. enterica & Infantis & Reference strain & - & - & - & + \\
\hline SARB 56 & S. enterica & Saintpaul & Reference strain & - & - & - & + \\
\hline SARB 63 & S. enterica & Typhi & Reference strain & - & - & - & + \\
\hline SARB 5 & S. enterica & Choleraesuis & Reference strain & - & - & - & + \\
\hline SARB 22 & S. enterica & Haifa & Reference strain & - & - & - & + \\
\hline Gram-positive control & & & & & & & \\
\hline NCTC 10239 & Clostridium perfringens & Gram positive & Reference strain & - & - & - & - \\
\hline
\end{tabular}

${ }^{a} \mathrm{ND}$, not determined; ETEEC, enterotoxemic E. coli; VTEC, verocytotoxigenic E. coli; EPEC, enteropathogenic E. coli; ETEC, enterotoxigenic $E$. coli. 
O16:K1:H showed the same pattern as WG1, E. coli serovar O17:K16:H18 and all other non-K-12 strains showed no bands with the primers derived from orf 264 . This confirms the close relationship of K-12 rfb wild-type genes with those of $E$. coli serovar O16. It implies that the rhamnose transferase is correlated with the expression of a specific $\mathrm{O}$ antigen. In addition, our results show that the gene orf 264 is present only in E. coli $\mathrm{K}-12$ and its derivatives and in serovar O16:K1:H. It is not detected in other E. coli, Salmonella, and Shigella strains isolated from a broad range of origins including humans, animals, and the environment.

Differentiation of a side lineage of K-12 derivatives. Interestingly, two K-12 derivative strains, AB311 and YN2980, showed no amplification product in the PCR with the primers K12-LK12-R flanking the IS5 insertion in orf264. Since both AB311 and YN2980 did amplify the right-handed junction of IS5 and orf 264 in the specific reaction for K-12 derivatives with the primer pair K12IS-L-K12-R, it seems that these two K-12 derivative strains have lost the segment of orf264 upstream of the site of integration of IS5 and therefore present a particular side lineage of K-12 derivatives which must have developed during successive mutagenesis. Moreover, we speculate that strain YN2980, which is not in the pedigree, is a descendant of AB311. Strains AB284 and W208, which are ancestors of AB311 (1), do not contain this deletion.

In summary, we have developed a PCR-based method which allows a rapid and accurate identification of E. coli $\mathrm{K}-12$ derivative strains. A single PCR with the primer pair K12IS-LK12-R amplifying the junction of the IS5 insertion in the rhamnose transferase gene (orf264) is sufficient for their positive identification. This insertion is expected to be highly specific to $\mathrm{K}-12$ derivative strains, since the orf264 gene was found only in K-12 strains and serovar O16, and IS5 was shown to be infrequent in natural $E$. coli isolates, to transpose rarely, and to integrate in various chromosomal loci (4).

Our analysis of 90 different E. coli strains from most different sources and 29 Salmonella and Shigella isolates showed that the method reliably identified all $39 \mathrm{~K}-12$ derivative strains and excluded all other E. coli, Salmonella, and Shigella strains, in spite of the fact that some of them are apparently very similar to the K-12 derivatives.

We thank Barbara J. Bachmann for sending us reference strains from the CGSC, A. Metzler and M. Frosch for supplying us with environmental isolates, L. Caro for sending us his collection strains, A. Burnens for help with pathogenic strains, P. Peveri for careful revision of the manuscript, and M. Küenzi (Basel, Switzerland) for stimulating discussions.

This work was supported by the Priority Program Biotechnology of the Swiss National Science Foundation (grant no. 5002-038920).

\section{REFERENCES}

1. Bachmann, B. J. 1987. Derivations and genotypes of some mutant derivatives of Escherichia coli K-12, p. 1190-1219. In F. C. Neidhardt, J. L. Ingraham, K. B. Low, B. Magasanik, M. Schaechter, and H. E. Umbarger (ed.), Escherichia coli and Salmonella typhimurium: cellular and molecular biology. American Society for Microbiology, Washington, D.C.

2. Chen, R., and U. Henning. 1987. Nucleotide sequence of the gene for the peptidoglycan-associated lipoprotein of Escherichia coli K12. Eur. J. Biochem. 163:73-77.

3. Gray, C. H., and E. L. Tatum. 1944. X-ray induced growth factor requirements in bacteria. Proc. Natl. Acad. Sci. USA 30:404-410.

4. Green, L., R. D. Miller, D. E. Dykhuizen, and D. L. Hartl. 1984. Distribution of DNA insertion element IS5 in natural isolates of Escherichia coli. Proc. Natl. Acad. Sci. USA 81:4000-4004.

5. Liu, D., and P. R. Reeves. 1994. Escherichia coli K12 regains its O antigen. Microbiology 140:49-57.

6. Mühldorfer, I., and J. Hacker. 1994. Genetic aspects of Escherichia coli virulence. Microb. Pathog. 16:171-181.

7. Sambrook, J., E. F. Fritsch, and T. Maniatis. 1989. Molecular cloning: a laboratory manual, 2nd ed. Cold Spring Harbor Laboratory Press, Cold Spring Harbor, N.Y.

8. Smiley, B. K., and F. C. Minion. 1993. Enhanced readthrough of opa (UGA) stop codons and production of Mycoplasma pneumoniae P1 epitopes in Escherichia coli. Gene 134:33-40.

9. Smith, H. W. 1975 . Survival of orally administered E. coli K12 in alimentary tract of human. Nature (London) 255:500-502

10. Stevenson, G., B. Neal, D. Liu, M. Hobbs, N. H. Packer, M. Batley, J. W. Redmond, L. Lindquist, and P. Reeves. 1994. Structure of the O antigen of Escherichia coli K-12 and the sequence of its $r f b$ gene cluster. J. Bacteriol. 176:4144-4156.

11. Yao, Z., and M. A. Valvano. 1994. Genetic analysis of the O-specific lipopolysaccharide biosynthesis region $(r f b)$ of Escherichia coli K-12 W3110: identification of genes that confer group 6 specificity to Shigella flexneri serotypes Y and 4a. J. Bacteriol. 176:4133-4143. 